\title{
Hierarchical porous ceramics with multiple open pores from boehmite gel emulsions
}

\author{
Yu Zheng ( $\nabla$ zhengyus@163.com ) \\ Liaoning University of Science and Technology \\ Xudong Luo \\ University of Science and Technology Liaoning \\ Jiegang You \\ University of Science and Technology Liaoning \\ Ting Li \\ University of Science and Technology Liaoning \\ Qingdong Hou \\ University of Science and Technology Liaoning
}

\section{Rapid Communication}

Keywords: Emulsion, Hierarchical structure, Open pores, Specific surface area

Posted Date: March 18th, 2020

DOI: https://doi.org/10.21203/rs.3.rs-17681/v1

License: (c) (i) This work is licensed under a Creative Commons Attribution 4.0 International License. Read Full License

Version of Record: A version of this preprint was published at Journal of the American Ceramic Society on October 1st, 2020. See the published version at https://doi.org/10.1111/jace.17489. 


\title{
Hierarchical porous ceramics with multiple open pores from boehmite gel emulsions
}

\author{
Yu Zheng, Xudong Luo, Jiegang You, Ting Li, Qingdong Hou \\ School of Materials and Metallurgy, Liaoning University of Science and Technology, Anshan, \\ liaoning 114051, P. R. China
}

\begin{abstract}
Ceramic foams with highly porous microstructure play significant roles in increasing area, especially those with open channels and superior specific surface area. A simple and versatile route to prepare ceramic foams with open pores has been put forward in this research, via gelation of boehmite nanoparticles-assembled emulsions. Notably, hierarchical porous microstructure with open channels and uniform pore structure was prepared. High specific surface area up to $389.4 \mathrm{~m}^{2} / \mathrm{g}$ is attainable, making them excellent adsorption material, when combining the merit of hierarchical pore structure. Furthermore, lattice-shaped ceramics are prepared via direct ink printing gelled emulsion, displaying the potential of forming lightweight material with complex shape and designable macro-structure. The 3D printed foams exhibit multiple open pores, which cover the length scale from mm-scale, to $\mu \mathrm{m}$-scale and nm-scale, making them promising materials in several fields, including adsorption, catalysts supports, and filtrations, etc.
\end{abstract}

Key words: Emulsion; Hierarchical structure; Open pores; Specific surface area 


\section{Introduction}

Owing to rewards from the synergy effect of different levels of pores regardless of scales, shapes, connectivity or locations, hierarchical porous ceramics generally behave outstanding multi-functionalities including high selectivity, specific surface area, relative strength as well as rapid mass transport, which could be well tuned according to the practical demands and also perform excellent in the applications involving adsorbents, catalyst supports, drug delivery, filtration, energy storage and the like ${ }^{[1-3]}$. The pore structure can be recognized as close or open pores, depending on the interconnectivity between adjacent ${ }^{[4]}$. In general, porous ceramics dominated by closed pores could be preferred choices for thermal insulations or wave-transparent materials, while those with open pores can be arranged to catalysts or filters ${ }^{[5]}$. For catalysts, porous ceramics combining both open pores and high specific surface area, high-temperature and corrosion resistance are in urgent need nowadays, also bio-implants from biocompatible ceramics with open pores have drawn more attention in recent decades ${ }^{[6]}$.

Therefore, hierarchical porous ceramics occupied predominantly by open pores, in addition to possessing high specific surface area and precisely designed pore distributions at varied levels would be promising for the abovementioned fields. Among the common processing routes for introducing pores, i. g., partial sintering, sacrificial fugitives, replica templates, direct foaming, freeze casting as well as $3 \mathrm{D}$ printing methods ${ }^{[6-11]}$, replica templates technique is known for the obtain of interconnected open cells, which would be realized by the impregnation of precursor and produce exactly the same structure with initial porous materials. However, the existing templates from the nature or synthesis template constrains the achievement of $\mu \mathrm{m}$-sized open pores, and the elimination of 
templates would also import new defects. As an alternative and efficient approach for porous materials with high interconnectivity, high internal phase emulsions that are stabilized by colloidal ceramic particles have proved to be a low-cost, eco-friendly, versatile way to prepare highly porous ceramics, and successfully applied in various systems involving hydroxyapatite scaffolds, mullite, $\mathrm{ZrO}_{2}, \mathrm{Si}_{3} \mathrm{~N}_{4}$, etc ${ }^{[12-15]}$. Furthermore, Minas C. et al. put forward to fabricate hierarchical porous ceramics via 3D printing emulsions and foams, where the emulsions or foams template the micropores.

Nowadays, the development of new technologies in key areas from adsorption to catalyst support propose increasingly demands for new structural materials with improved performance ${ }^{[16]}$. These materials will not only have high porosity, but also open pore structure and large specific surface area. We herein propose a novel method to prepare porous $\mathrm{Al}_{2} \mathrm{O}_{3}$ with open pore structure and large specific surface area via gelation of oil-in-water $(\mathrm{o} / \mathrm{w})$ emulsions stabilized by boehmite nanoparticles for the first time. Such a strategy enables the production of porous ceramic with open channel, large specific surface area and great possibility of 3D shaping. The simplicity of such route and establishment of open channel structure with large specific surface area put forward in this paper has considerable application potential in catalysis, separation, tissue engineering fields.

\section{Experimental procedures}

In this work, boehmite powder was purchased from Hangzhou Zhihuajie Technology Co., Ltd., China. Short chain amphiphilic pentanoic acid was acquired from Sinopharm Chemical Reagent Co., Ltd., Beijing, China. Octane was purchased from Shanghai 
Aladdin Biochemical Technology Co., Ltd., China, which act as the oil phase. Firstly, to obtain homogeneous boehmite colloidal suspension with solid loading of 20 wt\%, magnetic stirring of boehmite powder and deionized water were carried out in water bathing of $80^{\circ} \mathrm{C}$ for $24 \mathrm{~h}$. Subsequently, $0.35 \mathrm{wt} \%$ pentanoic acid (based on the mass of boehmite) was added to the well-dispersed suspension in a hood, followed by magnetic stirring for another $1 \mathrm{~min}$. Then, octane (the volume ratio of water to octane is 1:6) was added into the above aqueous suspension along with stirring. After that, mechanical stirring at speed of $1000 \mathrm{rpm}$ for 3-5 min was conducted for the above mixture to prepare emulsions with tiny drops, followed by the addition of $5 \mathrm{~mol} / \mathrm{L} \mathrm{KOH}$ solution into emulsions little by little to trigger gelation by increasing the $\mathrm{pH}$ value. 3D printing of gel emulsions was realized by using Ultimaker printer via direct ink writing (DIW). Drying process of the obtained emulsions were performed in the environment of $25^{\circ} \mathrm{C}$ and $80 \%$ humidity. Finally, the dried samples were sintered in a furnace with heating rate of $3^{\circ} \mathrm{C}$ /min and a dwelling time of $2 \mathrm{~h}$.

Zeta potential of boehmite nanoparticles was measured by a zeta potential analyzer (CD-7020, Colloidal Dynamics Co., Ltd., Ponte Vedra Beach, FL, USA), by employing $5 \mathrm{wt} \%$ boehmite suspension. The size distribution of pores and open channel were measured by Nano Measurer software (invented by Fudan University, Shanghai, China). The phase composites of dried foams and sintered foams were detected by X-ray diffraction with $\mathrm{Cu} \mathrm{K} \alpha$ radiation (XRD, D8 ADVANCE, Bruker, Karlsruhe, Germany). BET specific surface area and mesoporous properties of as-prepared samples at diverse temperatures were tested using nitrogen adsorption measurements (ASIC-2 Quantachrome Instruments, USA). Boehmite nanoparticles were sonicated for 15-20 
mins and then deposited onto a copper electron microscope grid to observe its morphology using Transmission Electronic Microscopy (TEM, S-5500, Hitachi, Japan). The microstructure of the obtained foams was observed by scanning electron microscope (SEM, MERLIN VP Compact, Carl Zeiss, Jena, Germany). The adsorption equilibrium isotherm of methanal at room temperature $\left(25^{\circ} \mathrm{C}\right)$ was measured by Static Volume Adsorption Instrument (Belsorp-Max, Bel, Japan).

\section{Results and discussion}

Other than commonly chosen micro-scale raw particles, nanoparticles would make for the improvement of porosity and specific surface area meanwhile lowering the sintering temperature ${ }^{[17]}$. Boehmite nanoparticles were selected in this research with their morphology present in Fig. 1(a). The particle size shown in Fig. 1(b) with bimodal distributions indicates that individual particle exhibits size ranging from $4 \mathrm{~nm}$ to $10 \mathrm{~nm}$, while the higher size distribution from $25 \mathrm{~nm}$ to $250 \mathrm{~nm}$ corresponds to the size of agglomerate. In view of preparing stable particle-stabilized emulsions, the hydrophobic modification of initial particles is very critical. As illustrated in Fig. 1(c), the zeta potential of boehmite suspension is positive with a relatively high zeta potential value of $66 \mathrm{mV}$ at $\mathrm{pH}$ of 4.0 , which features strongly positive charge as a consequence of the protonation of hydroxyls ${ }^{[18]}$. Herein, pentanoic acid was selected to modify the surface of boehmite particles by absorbing on their cationic surface via electrostatic attraction effect, which render boehmite particles capacity to exist stably on the interface of oil/water after emulsification. Owing to the increasing $\mathrm{pH}$-induced gelation process, the modified boehmite particles would aggregate together and form network at the oil/water interface 
in the three-dimensional space. After the evaporation of both water and oil solvent, porous structure constructed by the connected particles could be generated, as well illustrated in Fig. 1(d).
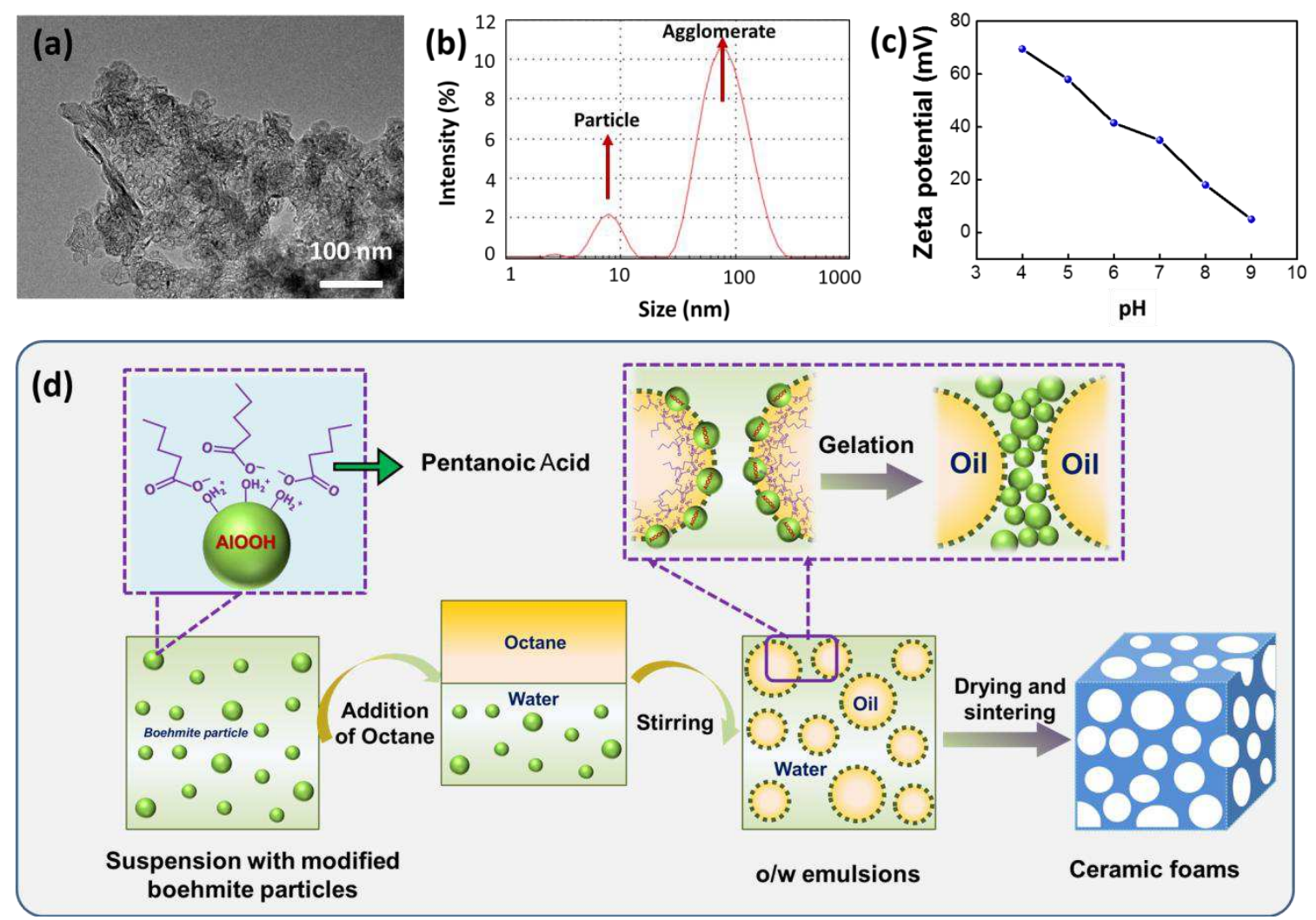

Fig. 1 (a) TEM image of boehmite particles, (b) particle size distribution of boehmite suspension and (c) zeta potential of $\mathrm{AlOOH}$ nanoparticles, (d) schematic representation depicting the formation of ceramic foams from emulsions.

Owing to the stable Pickering emulsions prepared via hydrophobic modification of boehmite particles as colloidal armor, and rapid gelation of emulsions, porous green bodies with uniform pore distribution were obtained after the consecutive drying process, as shown in Fig. 2(a). After sintering process, ceramic foams with uniform pore distribution as well as excellent interconnected pores were synthesized (see Fig. 2(b)), 
displaying well-defined open channels. The translucent position on the cell wall of cellular green body finally transforms to open windows after heat treatment, which could be well explained by the grain growth and phase transformation process and discussed later. In addition, the nanoscale boehmite particles assembled particle layer on the oil/water interface could account for the nanoscale pore wall of ceramic foams (see Fig. 2(c)), leading to the low bulk density of $0.106 \mathrm{~g} / \mathrm{cm}^{3}$. It is worth mentioning that the highly connected pores from emulsions as templates is a considerable alternative approach for open-cell ceramic foams due to the low-cost, simplicity, universality and eco-friendliness, having an advantage over the commonly used replica method which would lead to the microcracks from the template-removal process and cause problems like energy-consuming as well as environmental pollution ${ }^{[19]}$. 


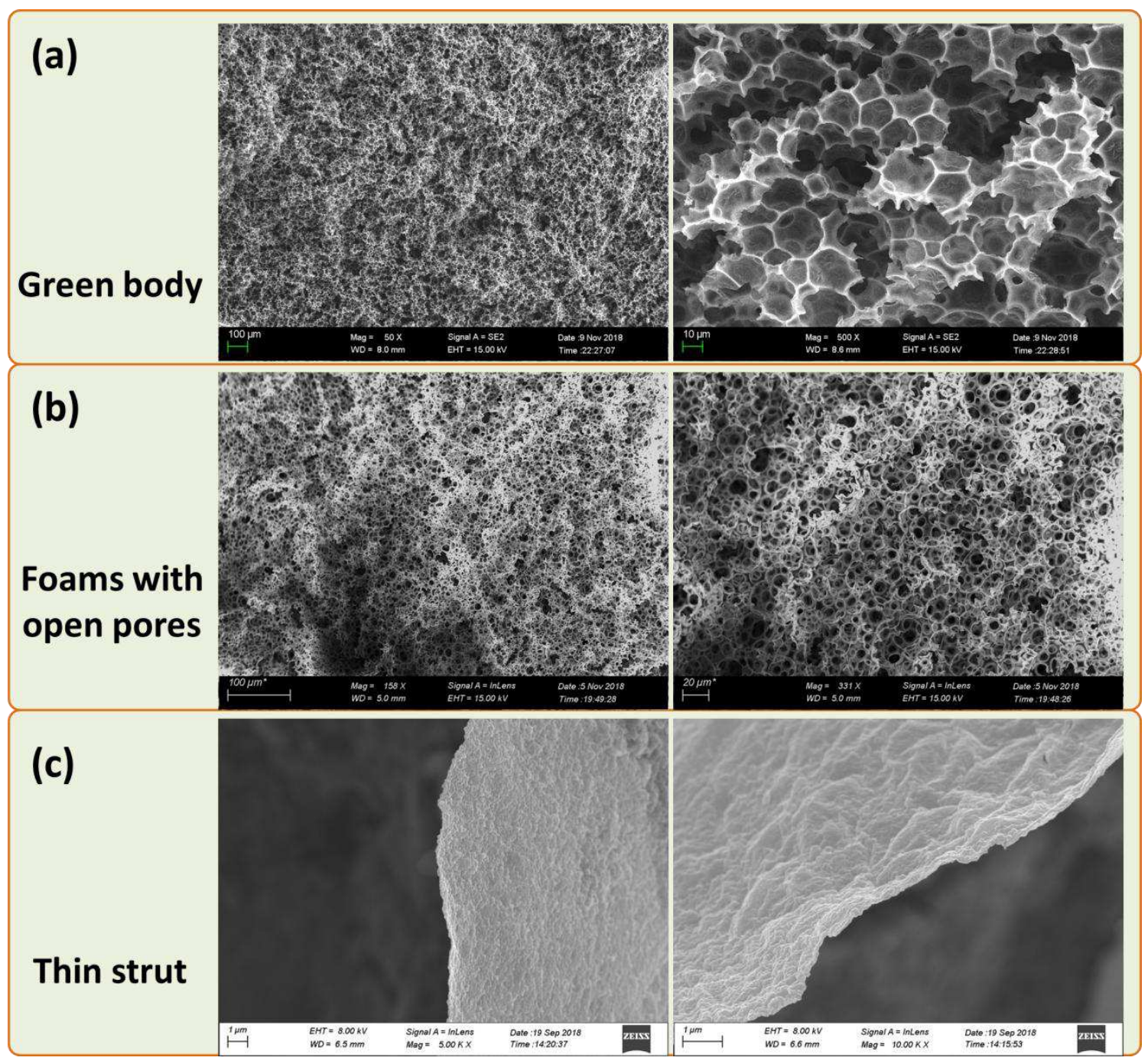

Fig. 2 SEM images of (a) green body and (b, c) ceramic foams sintered at $1000^{\circ} \mathrm{C}$

Attributed to the open channels derived from emulsions and the employment of nanoscale boehmite particles, hierarchical porous structure is fabricated in this work, as schematically illustrated in Fig. 3(a). Generally, three scale levels of pore size in the asprepared ceramic foams were fabricated, that is, first level of pores originated from oil droplets assembled by nanoparticles, second level of pores mainly caused by the densification of nanoparticles and the coalescence of inter-particle voids and the following grain growth process, and third level of pores derived from the residual gaps 
from the nanograins that forms mesoporous in the strut. The foam pore size ranges from $7.9 \mu \mathrm{m}$ to $27.2 \mu \mathrm{m}$ with an average pore size of $15.7 \mu \mathrm{m}$ (Fig. 3(b)). The open channels exhibit pore size ranging from $3.2 \mu \mathrm{m}$ to $9.3 \mu \mathrm{m}$, and the average pore size is $6.1 \mu \mathrm{m}$ (Fig. 3(c)). The mesoporous strut possesses average pore size around $20 \mathrm{~nm}$ (Fig. 3(d)).

(b)

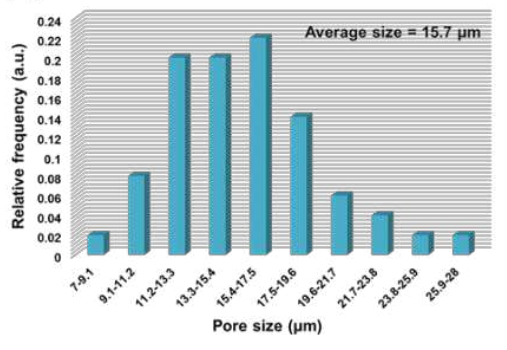

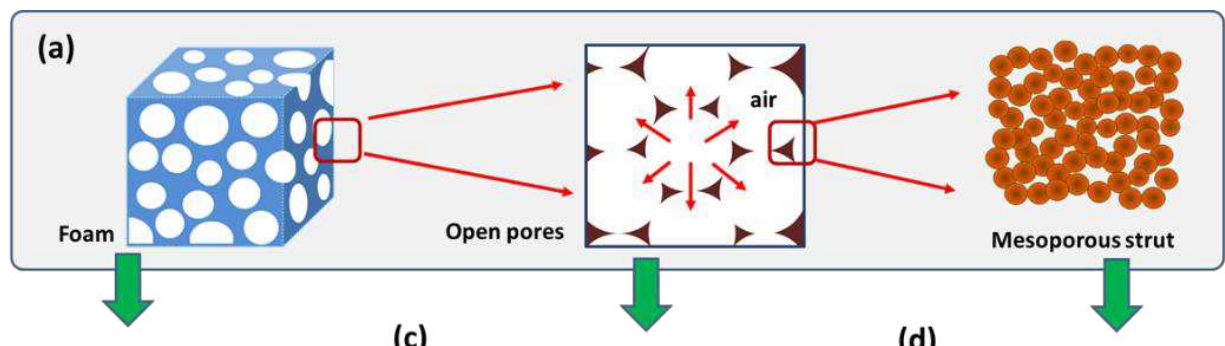

(c)

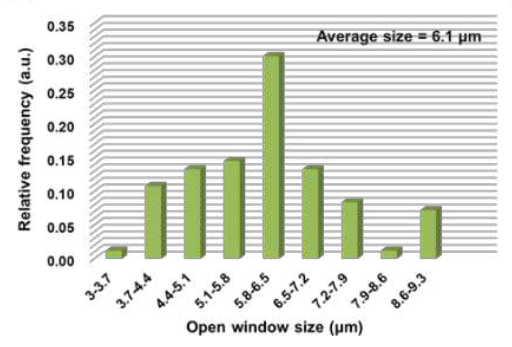

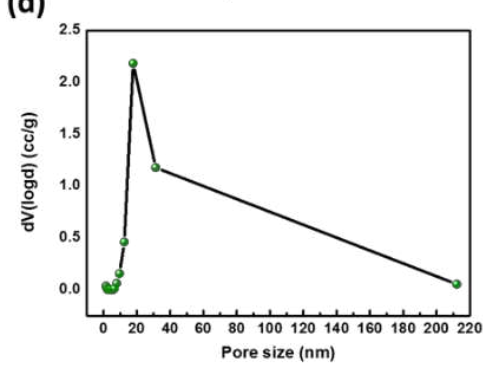

Fig. 3 (a) Schematic representation depicting the hierarchical structure in three length scales, pore size distribution of foam pores (b) open windows (c), and nanosized pores on strut (d)

XRD results states that the initial boehmite transforms into $\gamma-\mathrm{Al}_{2} \mathrm{O}_{3}$ when sintered at relatively low temperature of $400^{\circ} \mathrm{C}$ or $800^{\circ} \mathrm{C}$, and finally form $\alpha-\mathrm{Al}_{2} \mathrm{O}_{3}$ as the major phase accompanied by small amount of $\theta-\mathrm{Al}_{2} \mathrm{O}_{3}$ phase generated as the sintering temperature increase to $1200^{\circ} \mathrm{C}$. Notably, owing to the nanoparticles of as-received boehmite particles, the cellular green body present relatively high specific surface area of $362.6 \mathrm{~m}^{2} / \mathrm{g}$. Along with the increase of sintering temperature from $450^{\circ} \mathrm{C}$ to $1100^{\circ} \mathrm{C}$, the specific surface area of ceramic foams tends to decrease from $389.4 \mathrm{~m}^{2} / \mathrm{g}$ to $252.6 \mathrm{~m}^{2} / \mathrm{g}$, which is still equivalent to the reported aerogels ${ }^{[20-23]}$. Such kind of material with large 
specific surface area could supply accurate catalytic selectivity or reactive sites for guest matters, and facilitate the diffusion of gas or fluid through the interconnected skeleton. ${ }^{[16]}$ While further increasing the sintering temperature would have a negative influence on the specific surface area, and ceramic foams obtained at $1200^{\circ} \mathrm{C}$ only possess low specific surface area of $92.3 \mathrm{~m}^{2} / \mathrm{g}$, which could be attributed to the larger grains and reduced voids on the pore wall.

The as-prepared ceramic foams are featured by the interconnected pores that provide passages for the transportation of gas/liquid. The foams with high open channel and large specific surface area make them potential candidates for adsorption materials, as shown in Fig. 4(c). The maximum methanal adsorption amount is around $210 \mathrm{~cm}^{3} / \mathrm{g}$. Furthermore, we also demonstrate the possibility of 3D printing the gel emulsions into lattice-shape ceramics with multiple-levels open channels, large specific surface area, and low density, with the aid of direct ink writing method. As shown in Fig. 4(d), the macrostructure could be designed and constructed by the path of nozzles, which enable the formation of ceramic foams with complex shape on demand that could satisfy the gradually increasing needs in the catalysts, filters fields. 
(a)

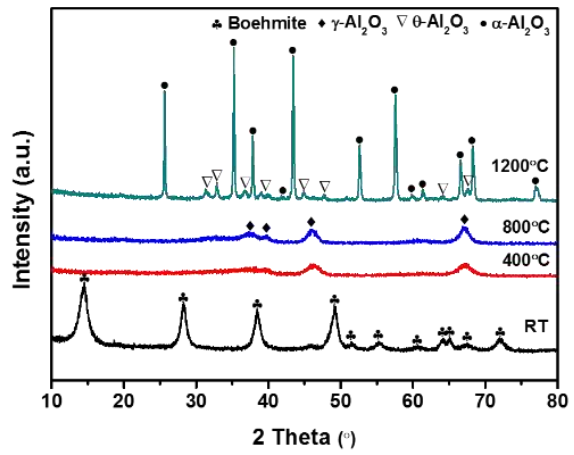

(c)

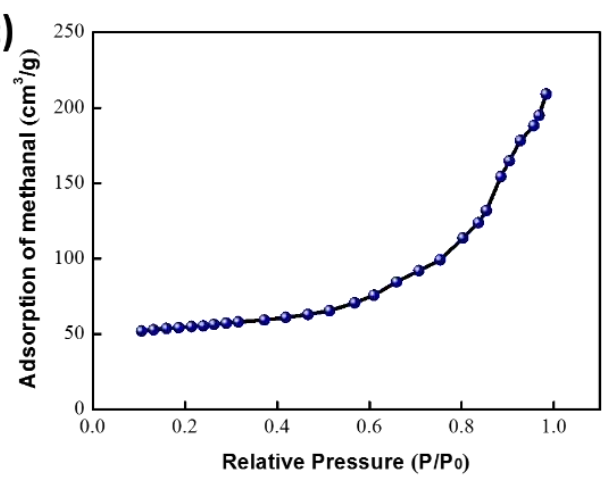

(b)

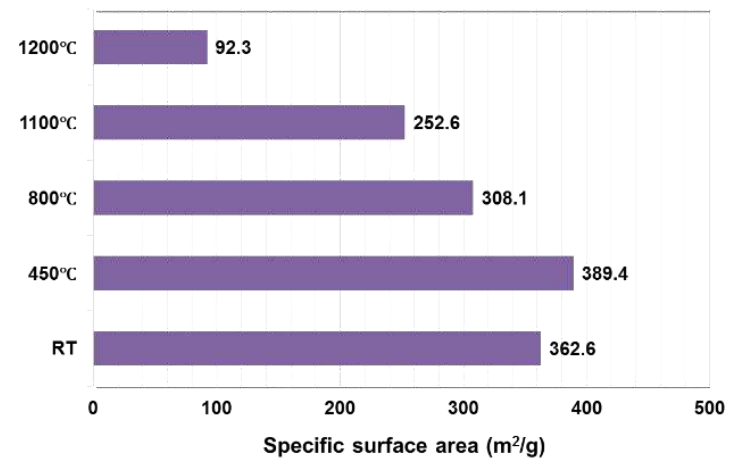

(d)

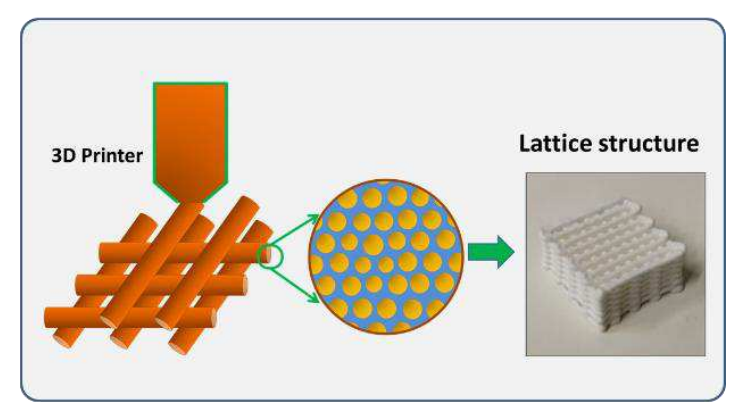

Fig. 4 (a) XRD patterns of foams sintered at various temperatures, (b) specific surface area of foams, (c) methanal adsorption curve of $800^{\circ} \mathrm{C}$ sintered foams, (d) 3D printing emulsions into lattice-shape sample with hierarchically open pore structure. 


\section{Conclusions}

The preparation of 3D hierarchical porous ceramics particularly with interconnected pores through gelation of particle-stabilized emulsions armored by boehmite nanoparticles are proposed here. The gelation via increasing the $\mathrm{pH}$ of suspension endows the gelled emulsions printability to conduct direct ink writing process, which enable the formation of ceramics with complex shape on demand. The hierarchical structure is featured by four levels of pore distribution, that is, filament-assembled structure, spherical pores from emulsion droplets as templates, open windows on the cell wall and nanoscale pores from the voids among grains. This highly interconnected ceramic foams possess high specific surface area up to $389.4 \mathrm{~m}^{2} / \mathrm{g}$, which is comparable to aerogels, and also show excellent capacity for the adsorption of methanal. The reported material with low bulk density, high interconnectivity, high specific surface area and outstanding adsorption capacity for organic solvent opens up the possibility of adsorptions, filtrations, catalyst supports, etc.

\section{Acknowledgments}

We are grateful for the financially support from the National Natural Science Foundation of China (Grant No. 51772139). 


\section{References}

[1] C. Minas, D. Carnelli, E. Tervoort, et al. 3D printing of emulsions and foams into hierarchical porous ceramics. Adv. Mater. 2016, 28: 9993-9999.

[2] C. Vakifahmetoglu, D. Zeydanli, V. C. Ozalp, et al. Hierarchically porous polymer derived ceramics: A promising platform for multidrug delivery systems. Mater. Des. 2018, 140: 37-44. [3] J. Han, L. Hu, Y. Zhang, et al. In situ synthesis of hierarchically porous silica ceramics with unidirectionally aligned channel structure. Scripta Mater. 2010, 62: 431-434.

[4] M. V. Twigg, J. T. Richardson. Fundamentals and applications of structured ceramic foam catalysts. Ind. Eng. Chem. Res. 2007, 46: 4166-4177.

[5] B. V. Manoj Kumar, Y. W. Kim. Processing of polysiloxane-derived porous ceramics: a review. Sci. Technol. Adv. Mat. 2010, 11: 044303.

[6] T. Ohji, M. Fukushima, Macro-porous ceramics: processing and properties. International Materials Reviews. 2012, 57(2): 115-131.

[7] K. Schwartzwalder, A. V. Somers. Methods of making porous ceramic articles. US Patent, No. $3090094,1963$.

[8] J. Zhou, J. Fan, G. Sun, et al. Preparation and properties of porous silicon nitride ceramics with uniform spherical pores by improved pore-forming agent method. J. Alloy. Compo. 2015, 632: 655-660.

[9] U. T. Gonzenbach, A. R. Studart, D. Steinlin, et al. Processing of particle-stabilized wet foams into porous ceramics. J. Am. Ceram. Soc. 2007, 90: 3407-3414.

[10] W. L. Li, K. Lu, J. Y. Walz. Freeze casting of porous materials: review of critical factors in microstructure evolution. Int. Mater. Rev. 2012, 57: 37-60.

[11] L. C. Hwa, S. Rajoo, A. M. Noor, et al. Recent advances in 3D printing of porous ceramics: A review. Curr. Opin. Solid St. M. 2017, 21: 323-347. 
[12] X. Wang, J. Li, Y. Xie, et al. Three-dimensional fully interconnected highly porous hydroxyapatite scaffolds derived from particle-stabilized emulsions. Ceram. Int. 2016, 42: 54555460.

[13] E.G. Moraes, P. Colombo, Silicon nitride foams from emulsions. Mater. Lett. 2014, 128 : 128-131.

[14] Y.A. Ivanova, C. Freitas, D.V. Lopes, et al. Cellular zirconia ceramics processed by direct emulsification, J. Eur. Ceram. Soc. 2020, 40: 2056-2062.

[15] X. Wang, J. Li, W. Guan, et al. Emulsion-templated high porosity mullite ceramics with sericite induced textured structures. Mater. Des. 2016, 89: 1041-1047.

[16] A. R. Studart, S. Julia, X. Lei, et al. Hierarchical porous materials made by drying complex suspensions. Langmuir. 2011, 27(3) 955-964.

[17] W. L. Huo, X. Y. Zhang, S. Y. Hou, et al. Aerogel - like ceramic foams with super - high porosity and nanoscale cell wall from sol nanoparticles stabilized foams. J. Am. Ceram. Soc. 2019, 102: 3753-3762.

[18] B. Tigges, T. Dederichs, M. Möller, et al. Interfacial Properties of Emulsions Stabilized with Surfactant and Nonsurfactant Coated Boehmite Nanoparticles. Langmuir 2010, 26:17913-17918.

[19] A. R. Studart, U. T. Gonzenbach, E. Tervoort, et al. Processing Routes to Macroporous Ceramics: A Review. J. Am. Ceram. Soc. 2006, 89: 1771-1789.

[20] G. Hayase, K. Nonomura, G. Hasegawa, et al. Ultralow-Density, Transparent, Superamphiphobic Boehmite Nanofiber Aerogels and Their Alumina Derivatives. Chem. Mater. 2015, 27: 3-5.

[21] J. F. Poco, J. H. S. Jr, L. W. Hrubesh. Synthesis of high porosity, monolithic alumina aerogels. J. Non-Cryst. Solids. 2001, 285:57 - 63.

[22] T. F. Baumann, A.E. Gash, S. C. Chinn, et al. Synthesis of high-surface-area alumina aerogels without the use of alkoxide precursors. Chem Mater. 2005,17:395-401. 
[23] G. Zu, J. Shen, X. Q. Wei, et al. Preparation and characterization of monolithic alumina aerogels. J. Non-Cryst. Solids. 2011, 357: 2903-2906. 
Figures
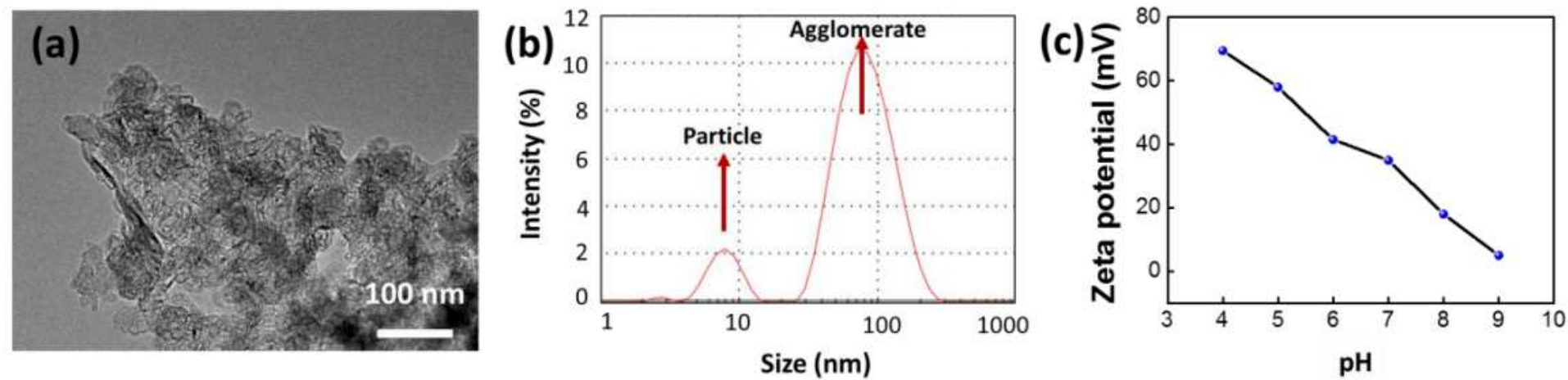

(d)

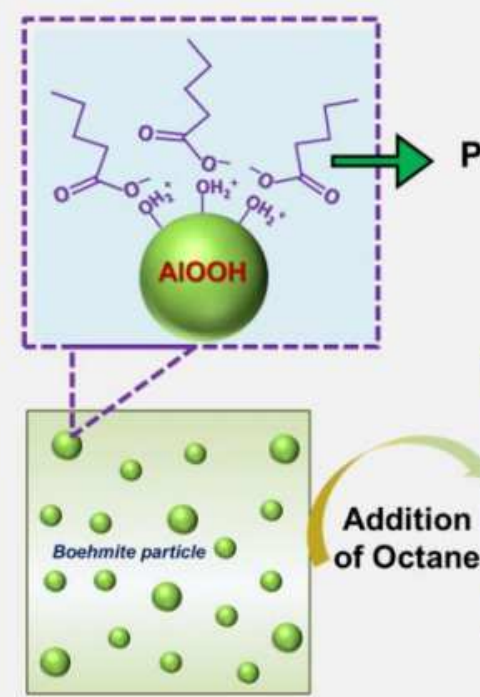

Pentanoic Acid

Suspension with modified boehmite particles

Figure 1

(a) TEM image of boehmite particles, (b) particle size distribution of boehmite suspension and (c) zeta potential of $\mathrm{AlOOH}$ nanoparticles, (d) schematic representation depicting the formation of ceramic foams from emulsions.

展

Figure 2

SEM images of (a) green body and (b, c) ceramic foams sintered at 1000区 
(b)

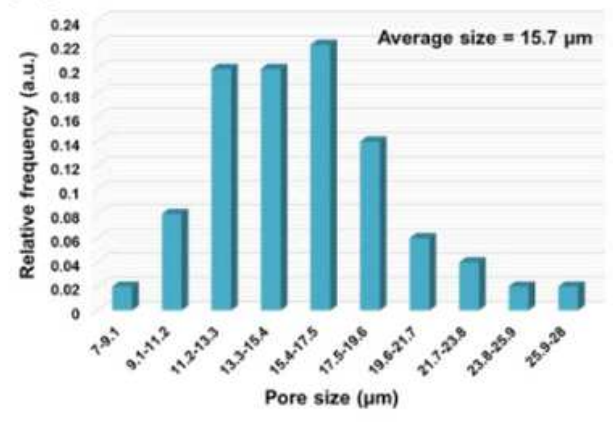

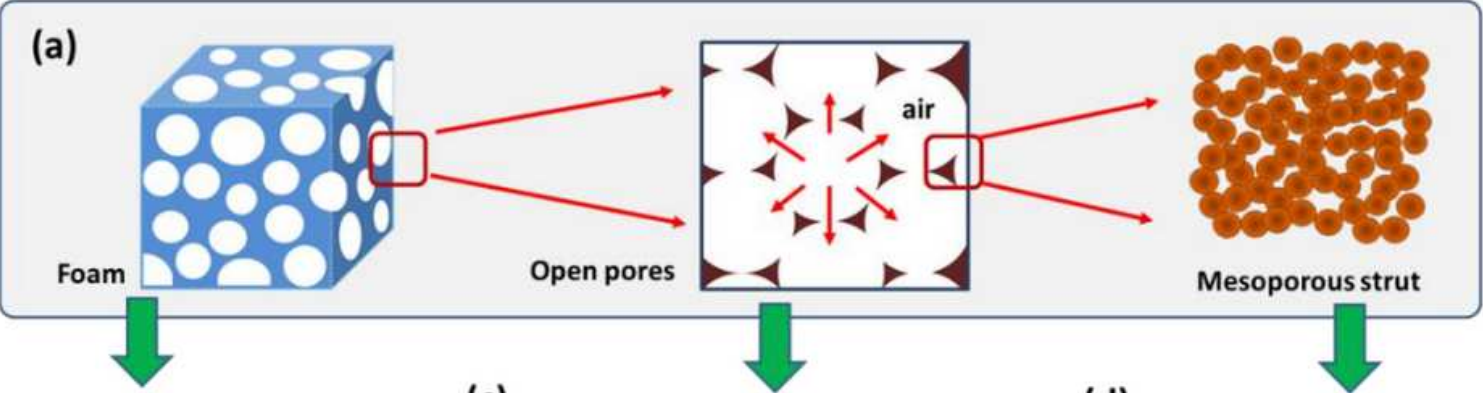

(c)

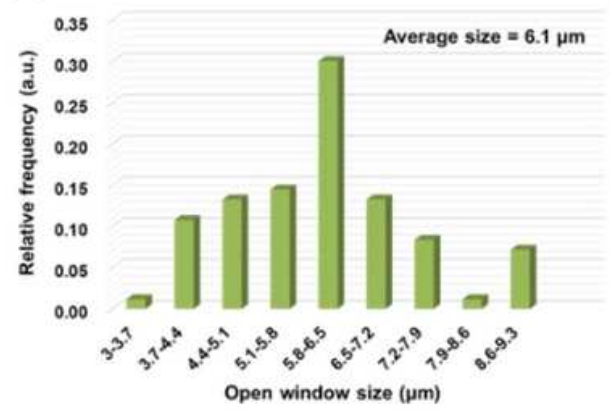

(d)

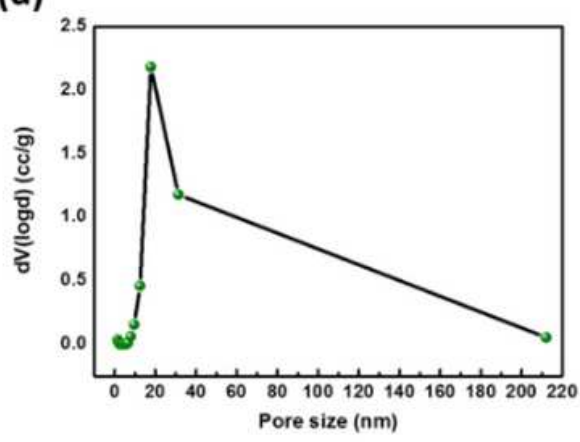

Figure 3

(a) Schematic representation depicting the hierarchical structure in three length scales, pore size distribution of foam pores (b) open windows (c), and nanosized pores on strut (d)

(a)

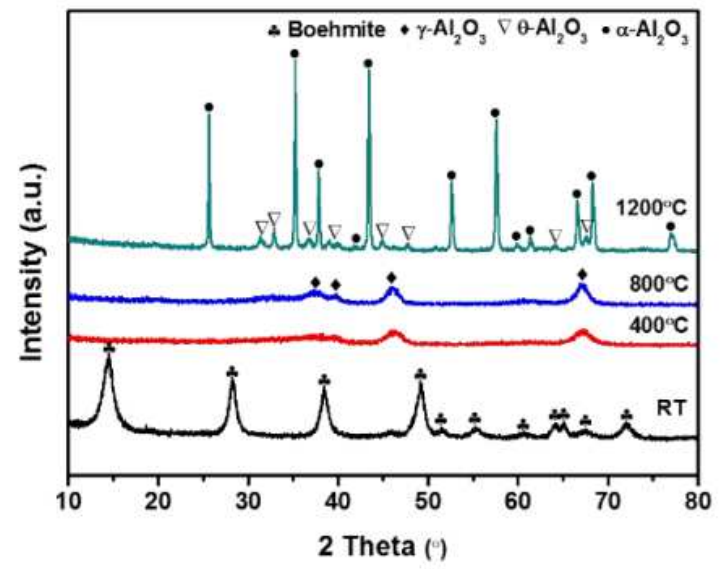

(c)

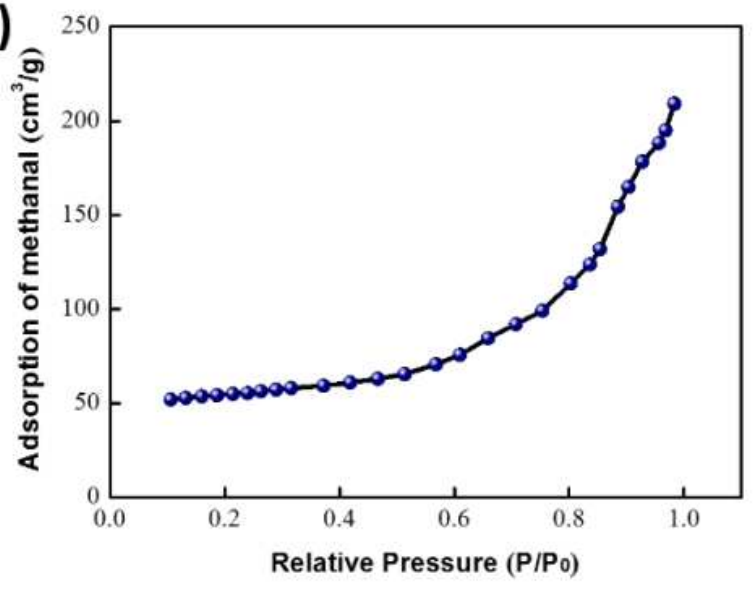

(b)

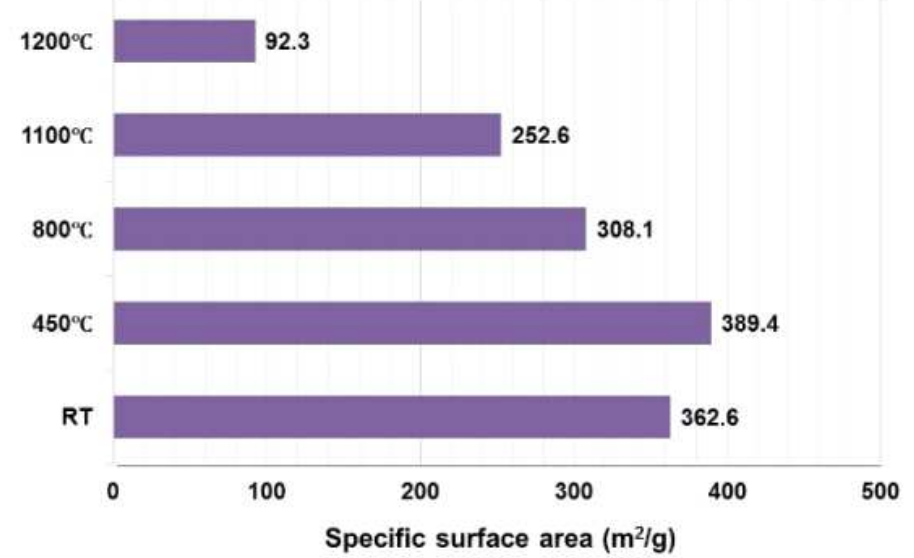

(d)

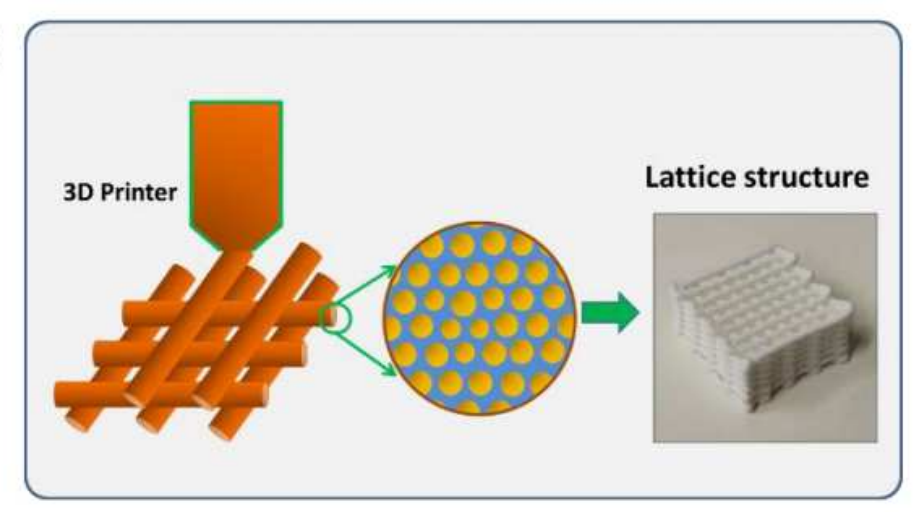




\section{Figure 4}

(a) XRD patterns of foams sintered at various temperatures, (b) specific surface area of foams, (c) methanal adsorption curve of $800^{\circ} \mathrm{C}$ sintered foams, (d) 3D printing emulsions into lattice-shape sample with hierarchically open pore structure. 